\title{
The Mathematics of Bitcoin
}

Cyril Grunspan (De Vinci Research Center, Pôle Léonard de Vinci, Courbevoie, France) and Ricardo Pérez-Marco (CNRS, IMJ-PRG, Sorbonne Université, Paris, France)

\section{Introduction to Bitcoin}

Bitcoin is a new decentralised payment network that started operating in January 2009. This new technology was created by a pseudonymous author, or group of authors, called Satoshi Nakamoto, in an article that was publically released [1] in the cypherpunk mailing list. The cypherpunks are anarchists and cryptographers who have been concerned with personal privacy in the Internet since the 90s. This article follows on from a general presentation of Bitcoin by the second author [2]. We refer to this previous article for general background. Here we focus on mathematics being a feature of the security and effectiveness of Bitcoin protocol.

Roughly speaking, the Bitcoin's protocol is a mathematical algorithm on a network which manages transaction data and builds majority consensus among the participants. Thus, if a majority of the participants are honest, then we get an honest automatic consensus. Its main feature is decentralisation, which means that no organisation or central structure is in charge. The nodes of the network are voluntary participants that enjoy equal rights and obligations. The network is open and anyone can participate. Once launched, the network is resilient and unstoppable. It has been functioning permanently without significant interruption since January 2009.

The code and development are open. The same code has been reused and modified to create hundreds of other cryptocurrencies based on the same principles. The security of the network relies on strong cryptography (several orders of magnitude stronger than the cryptography used in classical financial services). For example, classical hash functions (SHA256, RIPEMD-160) and elliptic curve digital signatures algorithm (ECDSA) are employed. The cryptography used is very standard and well known, so we will not dwell on the mathematics of these cryptographic tools, but interesting cryptographical research is motivated by the special features of other cryptocurrencies.

\section{Nodes and mining}

The bitcoin network is composed of nodes that correspond to the bitcoin program running on different machines and communicate with their neighbours. Properly formatted bitcoin transactions flood the network, and are checked, broadcasted and validated continuously by the nodes which follow a precise set of rules. There is no way to force the nodes to follow these rules. Incentives are created so that any divergence from the rules is economically penalised, thus creating a virtuous cycle. In this way, the network is a complex dynamical system and it is far from obvious that is is stable. The stability of this system is a very interesting and fundamental mathematical problem. In this study, we will encounter special functions, martingale theory, Markov chains, Dyck words, etc.

Nodes in the network broadcast transactions and can participate in their validation. The process of validating trans-

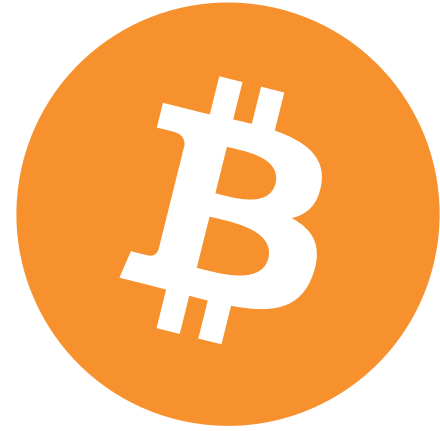

Figure 1. The bitcoin logo

actions is also called "mining" because it is related to the production of new bitcoins. The intuition behind bitcoin is that of a sort of "electronic gold", and the rate of production of bitcoins is implemented in the protocol rules. On average, every 10 minutes a block of transactions is validated and new bitcoins are minted in a special transaction without bitcoin input, called the coinbase transaction. At the beginning, $50 \mathrm{~B}$ were created in each block, and about every 4 years (more precisely, every 210.000 blocks), the production is divided by 2 . This event is called a "halving". So far, we have had two halvings, and the production is currently of $12.5 \mathrm{~B}$ per 10 minutes, or $1.800 \mathrm{~B}$ per day. The next halving will occur in April-May 2020. This geometric decrease of the production limits the total amount of bitcoins to 21 million. Currently, about 18 million have already been created. Each block containing the validated transactions can contain about 3 to 4 thousand transactions and has a size of about $1 \mathrm{Mb}$. These blocks are linked together cryptographically, and the set of all these blocks forms the "blockchain" that contains the full history of bitcoin transactions. This data is stored efficiently, and the current blockchain is only about $260.000 \mathrm{Mb}$. The cryptographical link between blocks is provided by the mining/validation procedure that is based on a hash function and a "Proof of Work". It costs computation power to validate a block and this is what ensures that the data cannot be tampered with or corrupted. In order to modify a single bit of a block, we must redo all computations that have been used to build all the subsequent blocks until the last current one. Currently the computation power needed to change the last few blocks of the more than 600 thousand composing the blockchain is beyond the capabilities of any state or company.

The mining/validation procedure is a sort of decentralised lottery. A miner (this is a node engaging in validating transactions) packs together a block of floating, not yet validated transactions, and builds a header of this block that contains a hash of the previous block header. The hash algorithm used is SHA-256 (iterated twice), that outputs 256 bits. Mathematically, a hash function is a deterministic one way function: it is easy to compute, but practically impossible to find pre-images or collisions (two files giving the same output). It also enjoys 
pseudo-random properties, that is, if we change a bit of the input, the bits of the output behave as uncorrelated random variables taking the values 0 and 1 with equal probabilities. The mining procedure consists of finding a hash that is below a pre-fixed threshold, which is called the difficulty. The difficulty is updated every two weeks (or more precisely every 2016 blocks) so that the rate of validation remains at 1 block per 10 minutes. The pseudo-random properties of the hash function ensure that the only way to find this hash is to probe many hashes, therefore changing a parameter in the header (the nonce). The first miner to find a solution makes the block public, and the network adopts the block as the last block in the blockchain.

It can happen that two blocks are simultaneously validated in different parts of the network. Then a competition follows between the two candidates, and the first one to have a mined block on top of it wins. The other one is discarded and is called an orphan block. The blockchain with the larger amount of work (which is in general the longer one) is adopted by the nodes.

When a transaction is included in the last block of the blockchain, we say that it has one confirmation. Any extra block mined on top of this one gives another confirmation to the transaction and engraves it further inside the blockchain.

This apparently complicated procedure is necessary to ensure that neither the network nor the blockchain can be corrupted. Any participant must commit some computer power in order to participate in the decision of validation. The main obstacle for the invention of a decentralised currency was to prevent a double spend without a central accounting authority. Hence, the first mathematical problem that Nakamoto considers in his founding article [1] is to estimate the probability of a double spend. In the following we consider this and other stability problems, and mathematically prove the (almost general) stability of the mining rules.

\section{$2 \quad$ The mining model}

We consider a miner with a fraction $0<p \leq 1$ of the total hashrate. His profit comes from the block rewards of his validated blocks. It is important to know the probability of success when mining a block. The average number of blocks per unit of time that he succeeds in mining is proportional to his hashrate $p$. The whole network takes on average $\tau_{0}=10 \mathrm{~min}$ to validate a block, hence our miner takes on average $t_{0}=\frac{\tau_{0}}{p}$. We consider the random variable $\mathbf{T}$ giving the time between blocks mined by our miner. The pseudo-random properties of the hash function show that mining is a Markov process, that is, memoryless. It is then an elementary exercise to show from this property that $\mathbf{T}$ follows an exponential distribution,

$$
f_{\tau}(t)=\alpha e^{-\alpha t}
$$

where $\alpha=1 / t_{0}=1 / \mathbb{E}[\mathbf{T}]$. If the miner starts mining at $t=0$, and if we denote $\mathbf{T}_{1}$ the time needed to mine a first block, then $\mathbf{T}_{2}, \ldots, \mathbf{T}_{n}$ the inter-block mining times of successive blocks, then the Markov property shows that the random variables $\mathbf{T}_{1}, \mathbf{T}_{2}, \ldots, \mathbf{T}_{n}$ are independent and are all identically distributed following the same exponential law. Therefore, the time needed to discover $n$ blocks is

$$
\mathbf{S}_{n}=\mathbf{T}_{1}+\mathbf{T}_{2}+\ldots+\mathbf{T}_{n} \text {. }
$$

The random variable $\mathbf{S}_{n}$ follows the $n$-convolution of the exponential distribution and, as is well known, this gives a Gamma distribution with parameters $(n, \alpha)$,

$$
f_{\mathbf{S}_{n}}(t)=\frac{\alpha^{n}}{(n-1) !} t^{n-1} e^{-\alpha t}
$$

with cumulative distribution

$$
F_{\mathbf{S}_{n}}(t)=\int_{0}^{t} f_{\mathbf{S}_{n}}(u) d u=1-e^{-\alpha t} \sum_{k=0}^{n-1} \frac{(\alpha t)^{k}}{k !} .
$$

From this we conclude that if $\mathbf{N}(t)$ is the process counting the number of blocks validated at time $t>0, \mathbf{N}(t)=\max \{n \geq$ $\left.0 ; \mathbf{S}_{n}<t\right\}$, then we have

$$
\mathbb{P}[\mathbf{N}(t)=n]=F_{\mathbf{S}_{n}}(t)-F_{\mathbf{S}_{n+1}}(t)=\frac{(\alpha t)^{n}}{n !} e^{-\alpha t},
$$

and $\mathbf{N}(t)$ follows a Poisson law with mean value $\alpha t$. This result is classical, and the mathematics of bitcoin mining, as well as other cryptocurrencies with validation based on proof of work, are mathematics of Poisson processes.

\section{The double spend problem}

The first crucial mathematical problem that deserves attention in the bitcoin protocol is the possibility of realisation of a double spend. This was the major obstacle to overcome for the invention of decentralised cryptocurrencies, thus it is not surprising that Nakamoto addresses this problem in Section 11 of his founding article [1]. He considers the situation where a malicious miner makes a payment, then in secret tries to validate a second conflicting transaction in a new block, from the same address, but to a new address that he controls, which allows him to recover the funds.

For this, once the first transaction has been validated in a block in the official blockchain and the vendor has delivered the goods (the vendor will not deliver unless some confirmations are visible), the only possibility consists of rewriting the blockchain from that block. This is feasible if he controls a majority of the hashrate, that is, if his relative hashrate $q$ satisfies $q>1 / 2$, because then he is able to mine faster than the rest of the network, and he can rewrite the last end of the blockchain as he desires. This is the reason why decentralised mining is necessary so that no one controls more than half of the mining power. But even when $0<q<1 / 2$ he can try to attempt a double spend and will succeed with a non-zero probability. The first mathematical problem consists of computing the probability that the malicious miner succeeds in rewriting the last $n \geq 1$ blocks. We assume that the remaining relative hashrate, $p=1-q$, consists of honest miners following the protocol rules.

This problem is similar to the classical gambler's ruin problem. Nakamoto observes that the probability of catching up $n$ blocks is

$$
q_{n}=\left(\frac{q}{p}\right)^{n}
$$

(Nakamoto)

The modelisation of mining shows that the processes $\mathbf{N}(t)$ and $\mathbf{N}^{\prime}(t)$ counting the number of mined blocks at time $t$ by the honest and malicious miners, respectively, are independent Poisson processes with respective parameters $\alpha$ et $\alpha^{\prime}$ satisfying

$$
p=\frac{\alpha}{\alpha+\alpha^{\prime}}, \quad q=\frac{\alpha^{\prime}}{\alpha+\alpha^{\prime}} .
$$


The random variable $\mathbf{X}_{n}=\mathbf{N}^{\prime}\left(\mathbf{S}_{n}\right)$ of the number of blocks mined by the attacker when the honest miners have mined their $n$-th block follows a negative binomial variable with parameters $(n, p)$ ([3]), thus, for an integer $k \geq 1$ we have

$$
\mathbb{P}\left[\mathbf{X}_{n}=k\right]=p^{k} q^{k}\left(\begin{array}{c}
k+n-1 \\
k
\end{array}\right) \text {. }
$$

Nakamoto, in section 11 of [1], abusively approximates $\mathbf{X}_{n}=$ $\mathbf{N}^{\prime}\left(\mathbf{S}_{n}\right)$ by $\mathbf{N}^{\prime}\left(t_{n}\right)$ where

$$
t_{n}=\mathbb{E}\left[\mathbf{S}_{n}\right]=n \mathbb{E}[\mathbf{T}]=\frac{n \tau_{0}}{p}
$$

This means that he considers the classical approximation of a negative binomial variable by a Poisson variable. Rosenfeld observes in [4] that the negative binomial variable seems to be a better approximation. We proved in [3] that this was indeed the case, and we could find the exact formula for the double spend probability after $z$ confirmations ( $z$ is the classical notation used by Nakamoto for the number of confirmations).

Theorem 1 ([3], 2017). After z confirmations, the probability of success of a double spend by attackers with a relative hashrate of $0<q<1 / 2$ is

$$
P(z)=I_{4 p q}(z, 1 / 2)
$$

where $I_{x}(a, b)$ is the incomplete regularised beta function

$$
I_{x}(a, b)=\frac{\Gamma(a+b)}{\Gamma(a) \Gamma(b)} \int_{0}^{x} t^{a-1}(1-t)^{b-1} d t .
$$

Bitcoin security depends on this probability computation. It is not just a theoretical result. It allows the estimation of the risk of a transaction to be reversed and the number of confirmations required to consider it definitive. For example, if $q=0.1$, after 6 confirmations, the probability of a double spend is smaller than $1 \%$ (for complete tables see [5]).

In his founding article, Nakamoto tried to compute this probability from his approximate argument and ran a numerical simulation. He convinced himself that the probability converges exponentially to 0 when the number of confirmations $z$ goes to infinite (as he states "we can see the probability drop off exponentially with $z$ "). The numerical simulation is not a proof, but this statement is repeated over and over again, however never proved before 2017. With the previous exact formula, using classical methods (Watson Lemma), we can prove the following Corollary:

Corollary 2. Let $s=4 p q<1$. When $z \rightarrow+\infty$ the probability $P(z)$ decays exponentially, and, more precisely,

$$
P(z) \sim \frac{s^{z}}{\sqrt{\pi(1-s) z}} .
$$

One can obtain higher order asymptotics in the classical way or by using equivalent combinatorical methods as in [6].

We can be more precise by looking at the time it takes for the honest network to mine $z$ blocks. A longer duration than the average $\tau_{1}=z \tau_{0}$ leaves extra time for the attacker to build his replacement blockchain, and with this conditional knowledge the probability changes. If we define $\kappa=\frac{\tau_{1}}{z \tau_{0}}$, we can compute this probability $P(z, \kappa)$ and we can also obtain an exact formula using the regularised incomplete Gamma function

$$
Q(s, x)=\frac{\Gamma(s, x)}{\Gamma(x)}
$$

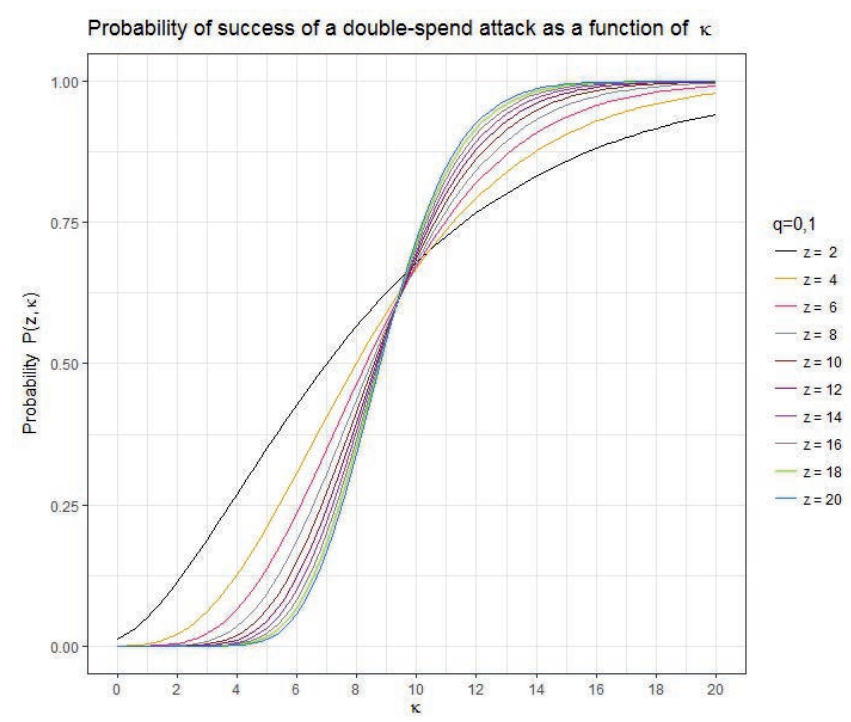

Figure 2. Probabilities $P(z, \kappa)$ for $q=0.1$ and distinct values of $z$

where

$$
\Gamma(s, x)=\int_{x}^{+\infty} t^{s-1} e^{-t} d t
$$

is the incomplete Gamma function.

Theorem 3. We have

$$
P(z, \kappa)=1-Q(z, \kappa z q / p)+\left(\frac{q}{p}\right)^{z} e^{\kappa z \frac{p-q}{p}} Q(z, \kappa z),
$$

Figure 2 shows the graphs of $\kappa \mapsto P(z, \kappa)$ for different values of $z$.

\section{$4 \quad$ Mining profitability}

After studying the security of the protocol, the next important problem is its stability. For a decentralised protocol it is fundamental that the interests of the individuals are properly aligned with the protocol rules. In particular, the maximal gain of miners should be achieved when following the protocol rules. This is far from obvious, and we know from the study of unstable dynamical systems that this is hard to achieve. It is somewhat surprising that this has been empirically verified since Bitcoin's inception.

For example, it is by no means obvious that is in the best interest of a miner to immediately publish a block that he has validated. He can keep it secret and secretly push his advantage, but then he runs the risk that another miner publishes a validated block and the public blockchain adopts it, thus losing his reward. This type of scenario has been discussed since 2012 in bitcointalk forum, created by Nakamoto in 2010.

To answer this question, we first need to develop a proper profitability model. As in any business, mining profitability is accounted by the "Profit and Loss" per unit of time. The profits of a miner come from the block rewards that include the coinbase reward in new bitcoins created, and the transaction fees of the transactions in the block. The profitability at 
instant $t>0$ is given by

$$
\mathbf{P L}(t)=\frac{\mathbf{R}(t)-\mathbf{C}(t)}{t}
$$

where $\mathbf{R}(t)$ and $\mathbf{C}(t)$ represent, respectively, the rewards and the cost of the mining operation up to time $t$. If we don't consider transaction fees, we have

$$
\mathbf{R}(t)=\mathbf{N}(t) b
$$

where $b>0$ is the coinbase reward. If we include transaction fees, the last equation remains true, taking the average reward using the classical Wald theorem.

The random variable $\mathbf{C}(t)$ representing the cost of mining operations is far more complex to determine, since it depends on external factors (such as electricity costs, mining hardware costs, geographic location, currency exchange rate, etc). But, fortunately, we don't need it in the comparison of the profitability of different mining strategies, as we explain next.

The mining activity is repetitive and the miners return to the same initial state after some time, for instance, to start mining a fresh block. A mining strategy is composed by cycles where the miner invariably returns to the initial state. It is a "game with repetition" similar to those employed by profit gamblers in casino games (when they can spot a weakness that makes the game profitable). For example, an honest miner starts a cycle each time the network, he or someone else, has validated a new block.

We denote by $\tau$ the duration of the cycle, and we are interested in integrable strategies for which $\mathbb{E}[\tau]<+\infty$ (this means that the cycles almost surely up in finite time). Then it is easy to check, using the law of large numbers and Wald theorem, that the long term profitability is given a.s. by the limit

$$
\mathbf{P L}_{\infty}=\lim _{t \rightarrow+\infty} \frac{\mathbf{R}(t)-\mathbf{C}(t)}{t}=\frac{\mathbb{E}[\mathbf{R}]-\mathbb{E}[\mathbf{C}]}{\mathbb{E}[\tau]} .
$$

As observed before, the second cost term is hard to compute, but the revenue term, that we call revenue ratio, is in general computable

$$
\Gamma=\frac{\mathbb{E}[\mathbf{R}]}{\mathbb{E}[\tau]} .
$$

For example, for an honest miner we have $\mathbb{E}[\mathbf{R}]=p \cdot 0+q \cdot b=$ $q b$ and $\mathbb{E}[\tau]=\tau_{0}$, and therefore

$$
\boldsymbol{\Gamma}_{H}=\frac{q b}{\tau_{0}} .
$$

We have the fundamental theorem on comparison of mining strategies with the same cost ratio. This is the case when both strategies use the full mining power at all time.

Theorem 4 ([7], 2018). We consider two mining strategies $\xi$ and $\eta$ with the same cost by unit of time. Then $\xi$ is more profitable than $\eta$ if and only if

$$
\boldsymbol{\Gamma}_{\eta} \leq \boldsymbol{\Gamma}_{\xi}
$$

\section{$5 \quad$ Protocol stability}

We can now mathematically study the protocol stability. The following remarkable result (remarkable because it is hard to imagine how Nakamoto could have foreseen it) validates the proper adjustment of the protocol:
Theorem 5 ([7], 2018). In the absence of difficulty adjustment, the optimal mining strategy is to immediately publish all mined blocks as soon as they are discovered.

Remember that the difficulty of mining adjusts about every two weeks, so at the same time we spot a weakness of the protocol that we discuss below.

This theorem holds true for any hashrate of the miner and without any assumption of the type of miners present in the network. It does not change anything that eventually there are some dishonest miners in the network.

The proof is simple and a good example of the power of martingale techniques. For a constant difficulty, the average speed of block discovery remains constant and the counting process $\mathbf{N}(t)$ is a Poisson process with intensity $\alpha=\frac{p}{\tau_{0}}$ where $p$ is the relative hashrate of the miner. The cycle duration $\tau$ is a stopping time and the revenue per cycle equals to $\mathbf{R}=\mathbf{N}(\tau)$. Its mean value is then obtained using Doob's stopping time to the martingale $\mathbf{N}(t)-\alpha t$. Finally, we get $\boldsymbol{\Gamma} \leq \boldsymbol{\Gamma}_{H}$.

But the bitcoin protocol does have a difficulty adjustment algorithm that is necessary, in particular during the development phase. Theorem 5 shows that this is the only vector of attack. This difficulty adjustment provides a steady monetary creation and ensures that the interblock validation time stays at around 10 minutes. A minimum delay is necessary to allow a good synchronisation of all network nodes. If the hashrate accelerates without a difficulty adjustment, then the nodes will desynchronize, and many competing blockchains will appear, leaving a chaotic state.

\section{$6 \quad$ Profitability of rogue strategies}

In view of Theorems 4 and 5, and in order to decide if a mining strategy is more profitable than the honest strategy, we need only compute the revenue ratio $\Gamma$ with the difficulty adjustment integrated. Selfish mining (SM strategy 1) is an example of rogue strategy. Instead of publishing a new block, the miner keeps the block secret and tries to build a longer blockchain, increasing its advantage. When he makes it public, he will orphan the last mined honest blocks and will reap the rewards. To be precise, the attack cycles are defined as follows: the miner starts mining a new block on top of the official blockchain. If an honest miner finds a block first, then the cycle ends and he starts over. Otherwise, when he is the first to find a block, he keeps mining on top of it, and keeping it secret. If before he mines a second block the honest network mines one public block, then he publishes his block immediately, thus trying to get a maximum proportion $0<\gamma<1$ of honest miners to adopt his block. The propagation is not instantaneous and the efficiency depends on the new parameter $\gamma$ which represents his good connectivity to the network. A competition follows, and if the next block is mined on top of the honest block, then the selfish miner looses the rewards of this block and the attack cycle ends. If he, or his allied honest miners, mine the next block, then they publish it and the attack cycle ends again. If the attacker succeeds in mining two consecutive secret blocks at the beginning, then he continues working on his secret blockchain until he has only one block of advantage with respect to the public blockchain. In this case, he doesn't run any risk of being joined by the pub- 
lic blockchain and publishes all his secret blockchain, thus reaping all the rewards and ending the attack cycle again. In few words, the rogue miner spends most of his time replacing honest blocks by those that he has mined in advance and kept secret. The mean duration $\mathbb{E}[\tau]$ of the attack cycle is obtained as a variation of the following result about Poisson processes.

Proposition 6 (Poisson races). Let $\mathbf{N}$ and $\mathbf{N}^{\prime}$ be two independent Poisson processes with respective parameters $\alpha$ and $\alpha^{\prime}$, with $\alpha^{\prime}<\alpha$ and $\mathbf{N}(0)=\mathbf{N}^{\prime}(0)=0$. Then, the stopping time

$$
\sigma=\inf \left\{t>0 ; \mathbf{N}(t)=\mathbf{N}^{\prime}(t)+1\right\}
$$

is almost surely finite, and we have

$$
\mathbb{E}[\sigma]=\frac{1}{\alpha-\alpha^{\prime}}, \quad \mathbb{E}\left[\mathbf{N}^{\prime}(\sigma)\right]=\frac{\alpha^{\prime}}{\alpha-\alpha^{\prime}}, \quad \mathbb{E}[\mathbf{N}(\sigma)]=\frac{\alpha}{\alpha-\alpha^{\prime}} .
$$

The proof is a simple application of Doob's stopping time theorem. Here, $\mathbf{N}$ and $\mathbf{N}^{\prime}$ are the counting processes of blocks mined by the honest miners and the attacker. To finish, we must compute the intensities $\alpha$ and $\alpha^{\prime}$. At the beginning we have $\alpha=\alpha_{0}=\frac{p}{\tau_{0}}$ and $\alpha^{\prime}=\alpha_{0}^{\prime}=\frac{q}{\tau_{0}}$, where $p$ is the apparent hashrate of the honest miners and $q$ the one of the attacker. But the existence of a selfish miner perturbs the network and slows down the production of blocks. Instead of having one block for each period $\tau_{0}$, the progression of the official blockchain is of $\mathbb{E}\left[N(\tau) \vee N^{\prime}(\tau)\right]$ blocks during $\mathbb{E}[\tau]$. After validation of 2016 official blocks, this triggers a difficulty adjustment that can be important. The new difficulty is obtained from the old one by multiplication by a factor $\delta<1$ given by

$$
\delta=\frac{\mathbb{E}\left[N(\boldsymbol{\tau}) \vee N^{\prime}(\tau)\right] \tau_{0}}{\mathbb{E}[\tau]} .
$$

After the difficulty adjustment, the new mining parameters are $\alpha=\alpha_{1}=\frac{\alpha_{0}}{\delta}$ and $\alpha^{\prime}=\alpha_{1}^{\prime}=\frac{\alpha_{0}^{\prime}}{\delta}$. The stopping time $\tau$ and the parameter $\delta$ can be computed using the relation $\mid N(\tau)-$ $N^{\prime}(\tau) \mid=1$. This can be used to compute the revenue ratio of the strategy [7]. This analysis can also be checked by mining simulators.

An alternative procedure consists of modelling the network by a Markov chain, where the different states correspond to a different degree of progress by the selfish miner. Each transition corresponds to a revenue increase $\pi$ and $\boldsymbol{\pi}^{\prime}$ for the honest and selfish miner. By another application of the law of large numbers, we prove that the long-term apparent hashrate of the strategy, defined as the proportion of mined blocks by the selfish miner compared to the total number of blocks, is given by the formula

$$
q^{\prime}=\frac{\mathbb{E}\left[\pi^{\prime}\right]}{\mathbb{E}[\pi]+\mathbb{E}\left[\pi^{\prime}\right]} .
$$

The expectation is taken as relative to the stationary probability that exists because the Markov chain is transitive and recurrent. Indeed, the Markov chain is essentially a random walk on $\mathbb{N}$ partially reflexive on 0 . The computation of this stationary probability proves the following theorem:

Theorem 7 ([8], 2014). The apparent hashrate of the selfish miner is

$$
q^{\prime}=\frac{((1+p q)(p-q)+p q) q-(1-\gamma) p^{2} q(p-q)}{p^{2} q+p-q}
$$

The results from [7] and [8] obtained by these different methods are compatible. The revenue ratio $\boldsymbol{\Gamma}_{1}$ and the apparent hashrate $q^{\prime}$ are related by the following equation:

$$
\boldsymbol{\Gamma}_{1}=q^{\prime} \frac{b}{\tau_{0}}
$$

But the first analysis is finer, since it does explain the change of profitablity regime after the difficulty adjustment. In particular, it allows us to compute the duration before running into profitability for the attacker. The selfish miner starts by losing money, then after the difficulty adjustment that favours him, starts making profits. For example, with $q=0.1$ and $\gamma=0.9$, he needs to wait 10 weeks in order to be profitable. This partly explains why such an attack has never been observed in the bitcoin network.

Theorem 4 gives an explicit semi-algebraic condition on the parameters, namely $q^{\prime}>q$, that determines the values of the parameters $q$ and $\gamma$ for which the selfish mining strategy is more profitable than honest mining.

Theorem 5 shows that the achilles heel of the protocole is the difficulty adjustment formula. This formula is supposed to contain the information about the total hashrate, but in reality it ignores the orphan blocks. The authors propose a solution that incorporates this count, and this solves the stability problem of the protocol [7].

There are other possible block-withholding strategies that are variations of the above strategy [9]. These are more agressive strategies. In the initial situation where the attacker succeeds in being two blocks ahead, instead of publishing the whole secret chain when he is only one block ahead, he can wait to be caught up to release his blocks and then start a final competition between the two competing chains. The attack cycle ends when the outcome is decided. This is the "Lead Stubborn Mining" (LSM, strategy 2). In this strategy it is important that the miner regularly publishes his secret blocks with the same height of the official blockchain, to attract part of the honest miners in order to take out hashrate from the pool of honest miners. Also in this way, even if he loses the final competition he will succeed in incorporating some of his blocks in the official blockchain and reap the corresponding rewards.

Another even more agressive variation consists of waiting not to be caught up, but to be behind one block. This is the "Equal Fork Stubborn Mining Strategy" (EFSM, strategy 3). Here again, it is important to publish secret blocks regularly. Finally, the authors have considered another more agressive variation where the stubborn miner follows EFSM but then doesn't stop when he is one block behind. He keeps on mining until his delay becomes greater than a threshold $A$ or until he successfully comes from behind, catches up and finally takes the advantage over the official blockchain.

This strategy seems desperate because the official blockchain progress is faster, on average. But in the case of catching up, the selfish miner wins the jackpot of all the blocks he replaces. This is the "A-Trailing Mining" strategy (A-TM, strategy 4). The authors of [9] conduct a numerical study of profitability by running a Montecarlo simulation and compare the profitability of the different strategies for different parameter values $(q, \gamma)$. But we can find closed form formulas for 


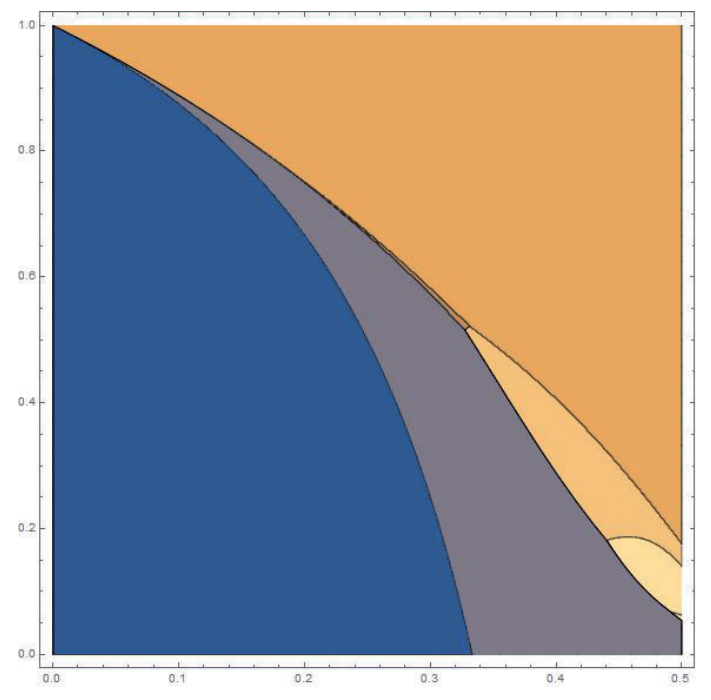

Figure 3. Comparison of HM, SM, LSM, EFSM and A-TSM

the revenue ratio of all these strategies using the precedent martingale approach.

Theorem 8 ([7, 10-12]). We have

$\frac{\boldsymbol{\Gamma}_{1}}{\boldsymbol{\Gamma}_{H}}=\frac{(1+p q)(p-q)+p q-(1-\gamma) p^{2}(p-q)}{p^{2} q+p-q}$

$\frac{\boldsymbol{\Gamma}_{2}}{\boldsymbol{\Gamma}_{H}}=\frac{p+p q-q^{2}}{p+p q-q}-\frac{p(p-q) f(\gamma, p, q)}{p+p q-q}$

$\frac{\boldsymbol{\Gamma}_{3}}{\boldsymbol{\Gamma}_{H}}=\frac{1}{p}-\frac{p-q}{p q} f(\gamma, p, q)$

$\frac{\boldsymbol{\Gamma}_{4}}{\boldsymbol{\Gamma}_{H}}$

$=\frac{1+\frac{(1-\gamma) p(p-q)}{\left(p+p q-q^{2}\right)[A+1]}\left(\left([A-1]+\frac{1}{p} \frac{P_{A}(\lambda)}{[A+1]}\right) \lambda^{2}-\frac{2}{\sqrt{1-4(1-\gamma) p q}+p-q}\right)}{\frac{p+p q-q}{p+p q-q^{2}}+\frac{(1-\gamma) p q}{p+p q-q^{2}}(A+\lambda)\left(\frac{1}{[A+1]}-\frac{1}{A+\lambda}\right)}$

with

$$
f(\gamma, p, q)=\frac{1-\gamma}{\gamma} \cdot\left(1-\frac{1}{2 q}(1-\sqrt{1-4(1-\gamma) p q})\right)
$$

and

$$
\begin{gathered}
\lambda=q / p, q \quad[n]=\frac{1-\lambda^{n}}{1-\lambda} \quad \text { for } n \in \mathbb{N}, \\
P_{A}(\lambda)=\frac{1-A \lambda^{A-1}+A \lambda^{A+1}-\lambda^{2 A}}{(1-\lambda)^{3}} .
\end{gathered}
$$

We can plot the parameter regions where each strategy is the best one (Figure 3). The Catalan numbers appear naturally in the computations.

$$
C_{n}=\frac{1}{2 n+1}\left(\begin{array}{c}
2 n \\
n
\end{array}\right)=\frac{(2 n) !}{n !(n+1) !} .
$$

For this reason, their generating function appears in the formulas

$$
C(x)=\sum_{n=0}^{+\infty} C_{n} x^{n}=\frac{1-\sqrt{1-4 x}}{2 x}
$$

We observe that $\sqrt{1-4 p q}=p-q$ and $C(p q)=1 / p$, and this justifies the definition of new probability distributions that arise in the proofs.
Definition 9. A discrete random variable $X$ taking integer values follows a Catalan distribution of the first type if we have, for $n \geq 0$,

$$
\mathbb{P}[X=n]=C_{n} p(p q)^{n} .
$$

It follows a Catalan distribution of the second type if $\mathbb{P}[X=$ $0]=p$ and for $n \geq 1$,

$$
\mathbb{P}[X=n]=C_{n-1}(p q)^{n} .
$$

It follows a Catalan distribution of the third type if $\mathbb{P}[X=0]=$ $p, \mathbb{P}[X=1]=p q+p q^{2}$ and for $n \geq 2$,

$$
\mathbb{P}[X=n]=p q^{2} C_{n-1}(p q)^{n-1} .
$$

\section{$7 \quad$ Dyck words}

We can recover these results by a direct combinatorical approach representing each attack cycle by a Dyck word.

Definition 10. A Dyck word is a word built from the two letter alphabet $\{S, H\}$ which contains as many $\mathrm{S}$ letters as $\mathrm{H}$ letters, and such that any prefix word contains more or equal $\mathrm{S}$ letters than $\mathrm{H}$ letters. We denote $\mathcal{D}$ the set of Dyck words, and for $n \geq 0, \mathcal{D}_{n}$ the subset of Dyck worlds of length $2 n$.

The relation to Catalan numbers is classical: the cardinal of $\mathcal{D}_{n}$ is $C_{n}$. We can encode attack cycles by a chronologic succession of block discoveries (disregarding if it is made public or not). For a selfish block we use the letter S (for "selfish") and for the honest blocks the letter H (for "honest").

The link between the selfish mining strategy and Dyck words is given by the following proposition:

Proposition 11. The attack cycles of the SM strategy are $H$, $S H H, S H S$, and $S S w H$ where $w \in \mathcal{D}$.

At the end of the cycle, we can summarise and count the total number of official blocks, say $L$, and how many of these blocks were mined by the attacker, say $Z$. Then, for strategy 1 (SM), the random variable $L-1$ follows a Catalan distribution of the third type, and except for some particular cases (when $L<3$ ), we always have $L=Z$. The apparent hashrate $q^{\prime}$ is then given by the formula:

$$
q^{\prime}=\frac{\mathbb{E}[Z]}{\mathbb{E}[L]}
$$

We can then directly recover Theorem 7 by this simpler combinatorical procedure [12]. The other rogue strategies can be studied in a similar way. The Catalan distribution of the first type arises in the study of the strategy EFSM (strategy 3), and the one of the second type for the strategy LSM (strategy 2). We can then recover all the results given by the Markov chain analysis. Unfortunately, we cannot recover the more finer results obtained by martingales techniques.

This sort of analysis applies to other Proof of Work cryptocurrencies, and to Ethereum, which has a more complex reward system and a different difficulty adjustment formula [13].

\section{$8 \quad$ Nakamoto double spend revisited}

We come back to the fundamental double spend problem from the Nakamoto bitcoin paper discussed in Section 3. In that 


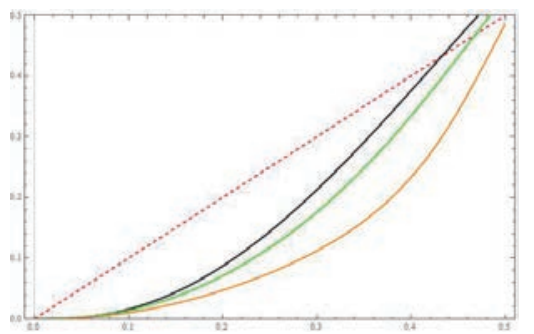

Figure 4. Graph of $\Gamma_{A}$, for $z=2, v=b$ and $A=3,5,10$

section, we computed the probability of success of a double spend. But now, with the profitability model knowledge from Section 4, we can study its profitability and get better estimates on the number of confirmations that are safe to consider a paiement definitive. The double spend strategy as presented in [1] is unsound because there is a non-zero probability of failure, and in that case, if we keep mining in the hope of catching up from far behind the official blockchain, we have a positive probability of total ruin. Also, the strategy is not integrable, since the expected duration of the attack is infinite. Thus, we must obviously apply a threshold to the unfavourable situation where we are lagging far behind the official blockchain.

We assume that the number of confirmations requested by the recipient of the transaction is $z$ and we assume that we are never behind $A \geq z$ blocks of the official blockchain. This defines an integrable strategy, the $A$-Nakamoto double spend strategy. Putting aside technical details about premining, the probability of success of this strategy is a modification of the probability from Theorem 1 .

Theorem 12 ([14], 2019). After $z$ confirmations, the probability of success of an A-Nakamoto double spend is

$$
P_{A}(z)=\frac{P(z)-\lambda^{A}}{1-\lambda^{A}}
$$

where $P(z)$ is the probability from Theorem 1 and $\lambda=q / p$.

If $v$ is the amount to double spend, then we can compute the revenue ratio $\boldsymbol{\Gamma}_{A}=\mathbb{E}[\mathbf{R}] / \mathbb{E}[\tau]$.

Theorem 13 ([14], 2019). With the previous notations, the expected revenue and the expected duration of the A-Nakamoto double spend strategy is

$$
\begin{aligned}
\mathbb{E}\left[\mathbf{R}_{A}\right] / b=\frac{q z}{2 p} & I_{4 p q}(z, 1 / 2)-\frac{A \lambda^{A}}{p(1-\lambda)^{3}[A]^{2}} I_{(p-q)^{2}}(1 / 2, z) \\
& +\frac{2-\lambda+\lambda^{A+1}}{(1-\lambda)^{2}[A]} \frac{p^{z-1} q^{z}}{B(z, z)}+P_{A}(z)\left(\frac{v}{b}+1\right) \\
\mathbb{E}\left[\mathbf{T}_{A}\right] / \tau_{0}= & \frac{z}{2 p} I_{4 p q}(z, 1 / 2)+\frac{A}{p(1-\lambda)^{2}[A]} I_{(p-q)^{2}}(1 / 2, z) \\
& -\frac{p^{z-1} q^{z}}{p(1-\lambda) B(z, z)}+\frac{1}{q}
\end{aligned}
$$

with the notation $[n]=\frac{1-\lambda^{n}}{1-\lambda}$ for an integer $n \geq 0$, and $B$ is the classical Beta function.

In principle, a powerful miner does not have an interest in participating in a large double spend, since doing so will undermine the foundations of his business. For a small miner with relative hashrate $0<q<<1$ we can estimate from which amount a double spend can be profitable. For this we only need to use the inequality from Theorem $4: \boldsymbol{\Gamma}_{A} \geq \boldsymbol{\Gamma}_{H}=q b / \tau_{0}$, and take the asymptotics $q \rightarrow 0$ (with $A$ and $z$ being fixed, but the final result turns out to be independent of $A$ ).

Corollary 14. When $q \rightarrow 0$, the minimal amount $v$ for an Nakamoto double spend with $z \geq 1$ confirmations is

$$
v \geq \frac{q^{-z}}{2\left(\begin{array}{c}
2 z-1 \\
z
\end{array}\right)} b=v_{0} .
$$

For example, in practice, with a $10 \%$ hashrate, $q=0.01$, and only one confirmation, $z=1$, we need to double spend more than $v_{0} / b=50$ coinbases. With the actual coinbase reward of $b=12.5 \mathrm{~B}$ and the actual prize over 8.300 euros, this represents more than 5 million euros.

Hence, for all practical purposes and normal amount transactions, only one confirmation is enough to consider the transaction definitive.

\section{Conclusions}

Bitcoin provides a good example of the universality of mathematical applications and its potential to impact our society. With the glimpse we have given, we hope to have convinced our colleagues that the Bitcoin protocol also motivates some exciting Mathematics.

\section{Bibliography}

[1] S. Nakamoto, "Bitcoin: A peer-to-peer electronic cash system," www.bitcoin.org/bitcoin.pdf, released on November 1st 2008 on the USENET Cryptography Mailing List "Bitcoin P2P e-cash paper".

[2] R. Pérez-Marco, "Bitcoin and decentralized trust protocols," Newsletter European Mathematical Society, vol. 21, no. 100, pp. 31-38, 2016.

[3] C. Grunspan and R. Pérez-Marco, "Double spend races," Int. Journal Theoretical and Applied Finance, vol. 21, no. 08, 2018.

[4] M. Rosenfeld, "Analysis of hashrate-based double spending," arXiv:1402.2009, 2014.

[5] C. Grunspan and R. Pérez-Marco, "Satoshi risk tables," arXiv:1702.04421, 2017.

[6] E. Giogladis and D. Zeilberger, "A combinatorial-probabilistic analysis of bitcoin attacks," Journal of Difference Equations and its Applications, vol. 25, no. 1, 2019.

[7] C. Grunspan and R. Pérez-Marco, "On the profitability of selfish mining," arXiv:1805.08281, 2018.

[8] I. Eyal and E. G. Sirer, "Majority is not enough: Bitcoin mining is vulnerable," Commun. ACM, vol. 61, pp. 95-102, June 2018.

[9] K. Nayak, S. Kumar, A. K. Miller, and E. Shi, "Stubborn mining: Generalizing selfish mining and combining with an eclipse attack," 2016 IEEE European Symposium on Security and Privacy (EuroSE P), pp. 305-320, 2015.

[10] C. Grunspan and R. Pérez-Marco, "On the profitability of stubborn mining," arXiv:1808.01041, 2018.

[11] C. Grunspan and R. Pérez-Marco, "On the profitability of trailing mining," arXiv:1811.09322, 2018.

[12] C. Grunspan and R. Pérez-Marco, "Bitcoin selfish mining and Dyck words," arXiv:1811.09322, 2019.

[13] C. Grunspan and R. Pérez-Marco, "Selfish mining in Ethereum," arXiv:1904.13330, 2019.

[14] C. Grunspan and R. Pérez-Marco, "On profitability of nakamoto double spend,” arXiv:1912.06412, 2019

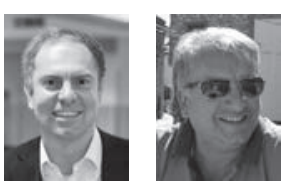

The authors [cyril@grunspan.net, ricardo.perez.marco@gmail.com] did study at Lycée Louis-le-Grand with the late Prof André Warusfel, and are alumni of the École Normale Supérieure of Paris. They are pioneers in mathematical research in Bitcoin and other cryptocurrencies and organize regularly the Paris cryptofinance seminar since 2016. 